\title{
Binary white dwarfs and decihertz gravitational wave observations: From the Hubble constant to supernova astrophysics.
}

\author{
A. Maselli ${ }^{1}$, S. Marassi ${ }^{1}$, and M. Branchesi ${ }^{2}$ \\ 1 Dipartimento di Fisica, "Sapienza” Università di Roma \& Sezione INFN Roma1, Piazzale Aldo Moro 5, 00185, Roma, Italy \\ e-mail: andrea.maselli@roma1.infn.it, e-mail: stefania.marassi@roma1.infn.it \\ ${ }^{2}$ Gran Sasso Science Institute (GSSI), I-67100 L'Aquila, Italy INFN, Laboratori Nazionali del Gran Sasso, I-67100 Assergi, Italy \\ e-mail: marica.branchesi@uniurb.it
}

February 4, 2020

\begin{abstract}
Context. Coalescences of binary white dwarfs represent a copious source of information for gravitational wave interferometers operating in the decihertz band. Moreover, according to the double degenerate scenario, they have been suggested to be possible progenitors of supernovae (SNe) Type Ia events.

Aims. In this paper we discuss the detectability of gravitational waves emitted by the inspiral of double white dwarfs. We focus on the constraints that can be derived on the source's luminosity distance, and on other binary's parameters, such as the angular momentum orientation.

Methods. We explore the possibility of coincident detections of gravitational and electromagnetic signals; the latter comes from the observation of the supernova counterpart. Confirmation of the double degenerate scenario would allow one to use distances inferred in the gravitational wave channel to consistently calibrate $\mathrm{SNe}$ as standard candles.

Results. We find that decihertz gravitational wave interferometers can measure the luminosity distance with relative accuracy better than $1 \%$ for binaries at $100 \mathrm{Mpc}$. We show how multimessenger observations can put strong constraints on the Hubble constant, which are tighter than current bounds at low redshift, and how they can potentially shed new light on the differences with early-universe measurements.
\end{abstract}

Key words. gravitational waves - binary white dwarf - Hubble constant

\section{Introduction}

Relativistic compact sources, such as black holes, neutron stars, and white dwarfs, represent natural laboratories to probe fundamental laws of physics (Barack et al. 2019). Gravitational wave (GW) observations of such objects have paved the way for a new understanding of the most extreme events of our Universe. (Abbott et al. 2016a b, 2017d e ff, 2018). These studies have aimed to address a large variety of open problems, which range from the status of matter at supranuclear density, to the nature of gravity in the strong field regime, and the dynamics of the expanding Universe (Sathyaprakash et al. 2019).

Among compact sources, binary white dwarfs (BWDs) feature a frequency content that is too low for the kilohertz sensitivity band of ground based detectors at the end of their orbital evolution. Conversely, BWDs will be sources of GWs in the millihertz regime for LISA, either as an unresolved background or as individually resolvable events (Nelemans et al. 2001). Moreover, BWDs represent a golden target for GW decihertz interferometers (Littenberg et al.2019), operating between 0.1 and 1 $\mathrm{Hz}$. These detectors can bridge the gap among existing and future ground-based facilities (Akutsu et al. 2019; Punturo et al. 2010; Dwyer et al. 2015; Abbott et al. 2017b) and the millihertz range spanned by LISA (Audley et al. 2017; McWilliams et al. 2019).

The GW decihertz interferometers represent unique laboratories to investigate the features of new astrophysical phenomena for the evolution of intermediate mass black holes (Yagi
2012), either in equal mass binaries or with a stellar companion, the stochastic background produced by cosmological sources (Marassi et al.2009; Kowalska et al.2012), or the nature of dark matter candidates (Kawamura et al. 2011). Moreover, they have been proposed as a new tool to exploit multiband GW observations of stellar mass sources (either black holes or neutron stars). They are able to provide precise measurements of the source's localization (Nair \& Tanaka 2018) and of the nuclear matter equation of state (Isoyama et al. 2018), and they allow for tests to be performed for gravity in the strong field regime (Carson \& Yagi 2019, Gnocchi et al. 2019).

Observations of BWDs by decihertz detectors would also shed new light on the evolutionary path of one of the most energetic events of our Universe, Type Ia supernovae (Mandel et al. 2018, Sedda et al. 2019). Currently, two possible scenarios are believed to provide a major explanation of such phenomena (Tutukov \& Yungelson 1981; Wang 2018) (see (Wang \& Han 2012, Maoz et al. 2014) for a recent review). In the so called "single degenerate scenario", WDs accrete from a main sequence or a giant companion and eventually reach the mass-threshold to explode (Whelan \& Iben 1973, Nielsen et al. 2014). According to the "double degenerate scenario", two WDs instead evolve through the emission of gravitational waves up to the merger phase, leading to the formation of single white dwarfs that are massive enough to ignite the burst (Webbink 1984, Iben \& Tutukov 1984, Raskin et al. 2012). So far, electromagnetic surveys have not been able to distinguish between these two cases (Rebassa-Mansergas et al. 2019). The GW signals in the deci- 
hertz regime can play a key role to probe nature and properties of the progenitors of supernovae ( $\mathrm{SNe}$ ) type Ia. In particular, coincident detections of SNe Ia and of GWs emitted by BWDs would provide the smoking gun needed to assess the consistency of the double degenerate scenario (Mandel et al. 2018; Sedda et al.2019).

Type Ia SNe also play a crucial role as cosmic ladders. Electromagnetic observations of their light curves and spectra allow one to determine the luminosity distance and the redshift and, therefore, we are able to determine the parameters of the underlying cosmological model and the local value of the Hubble constant, in particular (Hamuy et al. 1996; Riess et al. 1998; Kim et al. 1997). Current measurements of the Hubble constant can be classified into late-universe (Riess et al. 2019; Wong et al. 2019) and early-universe (Macaulay et al. 2019; Addison et al. 2018: Aghanim et al. 2018) estimates, which lead in turn to high and low values of $H_{0}$, respectively. The tension between these two classes may be traced back into uncontrolled systematics, or as the emergence of new physics in one, or both, of the two regimes (Riess et al. 2019; Aylor et al. 2019).

Gravitational waves have enabled us to potentially address this problem. Coalescing compact binaries represent pure standard sirens: The GW emitted by these systems do not require any calibration, and allow one to uniquely determine the luminosity distance of the source (Holz \& Hughes 2005, Dalal et al. 2006; Schutz 1986, Krolak \& Schutz 1987). Coincident observations of such events and of their electromagnetic counterparts would allow one to disentangle the distance information with the binary's redshift, providing the value of the Hubble constant and of the other cosmological parameters (Marković 1993; Finn \& Chernoff 1993). GW170817 and the associated electromagnetic counterpart represent a genuine example of multimessenger astrophysics that has already led to a first estimate of $H_{0}$ (Abbott et al. 2017f $|\mathrm{c}| \mathrm{a})$. Although the majority of GW measurements are not accurate enough to enable a direct identification of the host galaxy, and thus of the redshift, future samples of joint electromagnetic and gravitational-wave detections can resolve the Hubble tension (Nissanke et al. 2010, Chen et al. 2018; Feeney et al. 2019).

In this paper we study the gravitational wave emission by coalescing binary white dwarfs. We analyze the accuracy on the source parameters that can be derived by decihertz GW signals and the possibility to use joint supernovae Ia observations (within the double degenerate scenario) to improve such constraints.

We show how, in a multimessenger scenario, BWDs are a complementary and independent tool to measure $H_{0}$ and are able to provide new insights on the fundamental physics of the SN explosion. Gravitational signals are assumed to be observed by the Japanese decihertz interferometers DECIGO and B-DECIGO (Sato et al. 2017, Isoyama et al. 2018, Yagi \& Seto 2011). We focus on the constraints that these detectors will be able to derive on the luminosity distance and the Hubble constant, by exploiting the redshift inferred by the spectrum of SN or its host galaxy. Figure 1 shows a summary of our results for $H_{0}$ compared against some of the existing constraints. The bounds derived in this paper are tighter than those currently available from electromagnetic surveys at small $z$, and they are competitive with early Universe estimates that come, for example, from the cosmic microwave background (Aghanim et al. 2018). The precise measurements of the luminosity distance can also be used to consistently calibrate the supernova light curves, as recently proposed by Gupta et al. (2019) in the context of double neutron star mergers. We also show how when using GW signals emit- ted by BWDs, decihertz interferometers are able to accurately measure the inclination of the binary's orbital plane. The latter, together with the two component masses measured by GW emission, would provide a unique description of the binary's morphology, characterizing its full evolution, from the inspiral to the supernovae explosion. If not explicitly stated, throughout the paper we use geometrized units, in which $G=c=1$.

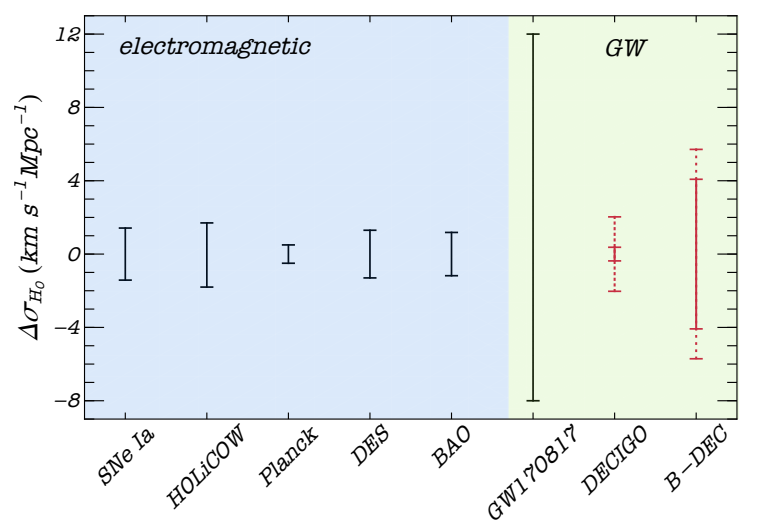

Fig. 1. 1- $\sigma$ interval on the local value of the Hubble constant $H_{0}$ obtained by different astrophysical observations in the electromagnetic and gravitational wave bands. We consider constraints derived by $\mathrm{SNe}$ Ia events (Riess et al. 2019), the HoliCOW experiment (Wong et al. 2019), the Planck mission (Aghanim et al. 2018), the Dark Energy Survey (Macaulay et al.2019), analysis of BAO (Addison et al.|2018), and the first measurement computed by coincident detection of the neutron star merger GW170817 and its EM counterpart (Abbott et al. 2017a). The last two values correspond to specific bounds derived in this work for binary WDs observed in the decihertz band by DECIGO and BDECIGO (see Table 1 2). Dashed and solid lines correspond to errors taking the correction due to peculiar velocity into account.

\section{Waveform's model}

We consider the orbital evolution of binary white dwarfs up to the merger phase. In the frequency domain, the GW signal emitted during the inspiral can be described by the following postNewtonian (PN) expanded GW waveform:

$\tilde{h}(f)=C_{\hat{\Omega}} \frac{\sqrt{3}}{2} \sqrt{\frac{5}{24}} \frac{G^{5 / 6} \mathcal{M}^{5 / 6}}{c^{3 / 2} \pi^{2 / 3} d} f^{-7 / 6} e^{i\left(\psi_{\mathrm{PN}}-\psi_{\mathrm{D}}-\psi_{\mathrm{pol}}\right)}$,

where $d$ is the luminosity distance of the binary, and $\left(t_{c}, \phi_{c}\right)$ is the time and phase at the coalescence. The PN phase $\psi_{\mathrm{PN}}$ depend ${ }^{1}$ on the chirp mass $\mathcal{M}=\left(m_{1} m_{2}\right)^{3 / 5} /\left(m_{1}+m_{2}\right)^{1 / 5}$ and on the symmetric mass ratio $v=\left(m_{1} m_{2}\right) /\left(m_{1}+m_{2}\right)^{2}$ (Khan et al. 2016), $\psi_{\mathrm{D}}$ describes the doppler shift of the phase wave front between the interferometer and the reference frame fixed with the Earth (or Sun) barycenter, and $\psi_{\text {pol }}$ is the polarization phase (Cutler 1998). The factor $C_{\hat{\Omega}}$ encodes the information on the source localization with respect to the detector's reference frame:

$C_{\hat{\Omega}}=\sqrt{F_{\times}^{2}(\hat{L} \cdot \hat{N})^{2}+F_{+}^{2}\left[1+(\hat{L} \cdot \hat{N})^{2}\right]^{2} / 4}$,

where $\hat{L} \cdot \hat{N}$ identifies the angle between the line of sight $\hat{N}$ and the binary's angular momentum $\hat{L}$, the latter being specified in

1 For the purposes of this work, we consider a 2 PN phase, which includes both the chirp mass and the symmetric mass ratio. We neglect spins and, in general, finite size effects of the WDs (Damour et al.2012. Vines et al. 2011). 
the orbital plane by the polar and azimuthal angles $\left(\bar{\theta}_{\mathrm{L}}, \bar{\phi}_{\mathrm{L}}\right)$. The interferometer's pattern functions $F_{\times,+}\left(\theta_{\mathrm{S}}, \phi_{\mathrm{S}}, \psi_{\mathrm{S}}\right)$ are defined as:

$F_{+}=\frac{1+\cos ^{2} \theta_{\mathrm{S}}}{2} \cos 2 \phi_{\mathrm{S}} \cos 2 \psi_{\mathrm{S}}-\cos \theta_{\mathrm{S}} \sin 2 \phi_{\mathrm{S}} \sin 2 \psi_{\mathrm{S}}$,

$F_{\times}=\frac{1+\cos ^{2} \theta_{\mathrm{S}}}{2} \cos 2 \phi_{\mathrm{S}} \sin \psi_{\mathrm{S}}+\cos \theta_{\mathrm{S}} \sin 2 \phi_{\mathrm{S}} \cos 2 \psi_{\mathrm{S}}$.

Here $\left(\theta_{\mathrm{S}}, \phi_{\mathrm{S}}\right)$ describe the location of the BWD in the sky and $\psi_{\mathrm{S}}$ is the polarization angle (Apostolatos et al. 1994), all of which are defined in the detector reference frame. The strong correlation between $d$ and the inclination of the binary makes it difficult to extract the luminosity distance of the source, in general. For ground based detectors, the lack of extra information coming from electromagnetic counterparts prevent precise measurements of $d$, even in case of multiple GW interferometers (Usman et al. 2019).

The three angles defined above, $\left(\theta_{\mathrm{S}}, \phi_{\mathrm{S}}, \psi_{\mathrm{S}}\right)$, actually depend on time, since the detector moves on the orbit around the Sun. When knowing the configuration of the interferometer with respect to the orbital plane, it is straightforward to express these quantities in terms of the BWD angular velocity and of constant angles $\left(\bar{\theta}_{\mathrm{S}}, \bar{\phi}_{\mathrm{S}}\right)$, which are defined in a fixed reference frame centered with the Sun (Cutler 1998; Yagi \& Seto 2011; Nair \& Tanaka 2018). We refer the reader to Appendix A for the full expression of time-dependent variables as a function of constant quantities. The template in eq. (1) therefore depends on nine parameters, namely $\lambda=\left(t_{c}, \phi_{c}, \ln \mathcal{M}, \ln v, d, \bar{\theta}_{\mathrm{S}}, \bar{\phi}_{\mathrm{S}}, \bar{\theta}_{\mathrm{L}}, \bar{\phi}_{\mathrm{L}}\right)$.

\subsection{Signal-to-noise ratio and errors}

Given the gravitational waveform (1), the signal-to-noise ratio $(\mathrm{S} / \mathrm{N})$ for a given detector is given by the noise weighted inner product of $\tilde{h}(f)$, that is:

$\rho^{2}=4 \operatorname{Re} \int_{f_{\min }}^{f_{\max }} \frac{d f}{S_{n}(f)}|\tilde{h}(f)|^{2}$,

with $S_{n}(f)$ being the detector's noise spectral density (Cutler 1998), which is explicitly given for DECIGO and B-DECIGO in Appendix A. For the systems considered in this work, we assume that $f_{\max }$ corresponds to the contact frequency of the system when the orbital separation is equal to $r_{\mathrm{WD}}\left(m_{1}\right)+r_{\mathrm{WD}}\left(m_{2}\right)$, assuming that the radius of the WD is given by the semi-analytic relation (Schneider et al. 2001, Portegies Zwart \& Verbunt|1996 Nauenberg 1972):

$\frac{r_{\mathrm{WD}}\left(m_{i}\right)}{R_{\odot}}=0.012\left[\left(\frac{m_{i}}{1.44 M_{\odot}}\right)^{-\frac{2}{3}}-\left(\frac{m_{i}}{1.44 M_{\odot}}\right)^{\frac{2}{3}}\right]^{1 / 2}, i=1,2$,

while $f_{\min }$ corresponds to the frequency $T=5$ years before the merger. Specifically, the time evolution of the GW radiation in terms of the frequency is given at the two PN order by:

$$
\begin{array}{r}
t(f)=t_{c}-\frac{5}{256} \mathcal{M}(\pi \mathcal{M} f)^{-8 / 3}\left[1+\frac{4}{3}\left(\frac{743}{336}+\frac{11}{4} v\right) x-\frac{32 \pi}{5} x^{3 / 2}\right. \\
\left.+2\left(\frac{3058673}{1016064}+\frac{5429}{1008} v+\frac{617}{144} v^{2}\right) x^{2}\right]
\end{array}
$$

where $x=(\pi M f)^{2 / 3}$ (Berti et al.2005). For a given set of masses and observing time $T$, the minimum frequency can be numerically found as the solution of $t\left(f_{\max }\right)-t\left(f_{\min }\right)=T$.
For high S/Ns, the statistical errors on the source parameters can be computed through the Fisher information matrix (Vallisneri 2008), defined as:

$\Gamma_{i j}=4 \operatorname{Re} \int_{f_{\min }}^{f_{\max }} \frac{d f}{S_{n}(f)} \frac{\partial \tilde{h}^{\star}(f)}{\partial \lambda^{i}} \frac{\partial \tilde{h}(f)}{\partial \lambda^{j}}$,

where $\tilde{h}^{\star}(f)$ is the complex conjugate of the waveform, $\lambda^{i}$ is the $i$-th term of the parameter's vector, and the Fisher is computed at the true values of $\lambda$. In this limit, the probability distribution of $\lambda$, for a given detector's output $s$ is proportional to $\Gamma_{i j}$, that is, $p(\lambda \mid s) \propto p_{0}(\lambda) \exp \left[-\frac{1}{2} \delta \lambda^{i} \Gamma_{i j} \delta \lambda^{i}\right]$, where $\delta \lambda^{i}$ is the measurement shift with respect to the real values and $p_{0}(\lambda)$ is our prior information on $\lambda$ (Poisson \& Will 1995). Diagonal and off-diagonal elements of the inverse of the Fisher matrix correspond to the root mean square and the correlation's coefficients of the source parameters:

$\sigma_{\lambda_{i}}=\sqrt{\left(\Gamma^{-1}\right)_{i i}} \quad, \quad C_{\lambda_{i}, \lambda_{j}}=\frac{\left(\Gamma^{-1}\right)_{i i}}{\sqrt{\sigma_{\lambda_{i}} \sigma_{\lambda_{j}}}}$.

Following (Yagi \& Seto 2011), we assume that each of the triangle units of (B-)DECIGO can be effectively considered as a system of two L-shaped interferometers, with the second being rotated $45^{\circ}$ with respect to the first one. In this configuration the pattern function of the second detector is given by $F_{\times,+}^{(2)}=$ $F_{\times,+}^{(1)}\left(\theta_{\mathrm{S}}, \phi_{\mathrm{S}}-\pi / 4, \psi_{\mathrm{S}}\right)$, and we can introduce a total $\mathrm{S} / \mathrm{N}$ of $\rho=$ $\sqrt{\rho_{(1)}^{2}+\rho_{(2)}^{2}}$. In the same way, the errors on the source parameters are obtained by inverting the sum of the Fisher matrices, that is, $\sigma_{\lambda_{i}}^{2}=\left(\Gamma^{(1)}+\Gamma^{(2)}\right)_{i i}$.

The last ingredient of our analysis is given by the prior information on $\lambda$. In this work we consider two possible scenarios: (i) BWDs that are only observed in the GW band, (ii) coincidence detections of WD mergers in both the electromagnetic and the gravitational spectrum. In this second scenario, we assume that the binary evolves according to the double degenerate scenario, igniting a SN Ia explosion. This assumption allows one to constrain the source's redshift $z$ and its sky position through the two angles $\left(\bar{\theta}_{\mathrm{S}}, \bar{\phi}_{\mathrm{S}}\right)$. We assume that the statistical errors on the polar and azimuthal angles are such that we can effectively reduce the dimensionality of the Fisher matrix and therefore of the parameter's vector to $\lambda=\left(t_{c}, \phi_{c}, \ln \mathcal{M}, \ln v, d, \bar{\theta}_{\mathrm{L}}, \bar{\phi}_{\mathrm{L}}\right)$.

It is important to note that, unlike short gamma ray bursts that are associated with neutron star mergers, supernova explosions are not expected to be beamed. Consequently, they do not provide any information on the inclination angle of the binary, that is, the values of $\bar{\theta}_{\mathrm{L}}$ and of $\bar{\phi}_{\mathrm{L}}$ (Abbott et al.2017c).

In a multimessenger scenario, given the error of the luminosity distance and the knowledge of $z$, it is straightforward to propagate the uncertainty of $H_{0}$. For small redshifts $z<0.05$ $(200 \mathrm{Mpc})$ for those considered in this paper, the local value of the Hubble constant can be determined by the Hubble-Lemaitre law, $H_{0} \simeq z / d$, neglecting errors ${ }^{2}$ on $z$ this yields $\sigma_{H_{0}} \simeq z \sigma_{d_{\mathrm{L}}} / d^{2}$. An additional source of uncertainty as to the $H_{0}$ measurement is given by the peculiar velocities; when the source is relatively close to the observer, the random relative motions of the galaxies due to gravitational interaction with nearby galaxies and overdensities are not negligible and the measured recessional velocity needs to be corrected in order to obtain the Hubble flow ve-

\footnotetext{
2 We assume that an uncertainty as to the redshift, measured by the supernova electromagnetic observations, is subleading compared to the other quantities in the analysis.
} 
locity. The unknown peculiar velocity is included in our $H_{0}$ uncertainties by adding in quadrature an additional statistical uncertainty of $1-\sigma=200 \mathrm{~km} / \mathrm{s}$, which is a typical uncertainty for the peculiar velocity correction (Carrick et al. 2015).

\section{Results}

As a first step, we have investigated the observational window spanned by the interferometers for BWD systems. The two panels of Fig. 2 show the contour lines of fixed S/Ns as a function of the stellar masses for prototype binaries with $m_{1,2} \in$ $[0.4,1.3] M_{\odot}$. Left and right plots correspond to systems observed by B-DECIGO and DECIGO at luminosity distances of $d=50 \mathrm{Mpc}$ and $d=100 \mathrm{Mpc}$, respectively. We also averaged the GW signals over the sky localization and the polarization angles, knowing that $\left\langle C_{\hat{\Omega}}^{2}\right\rangle=4 / 25$. The shaded gray delimits the region where the two WD could lead to a SN type Ia event. For both the interferometers, a large portion of the parameter space does exist in which the binaries will be observed with a $\mathrm{S} / \mathrm{N}$ above a threshold that, hereafter, we set to eight. For example, B-DECIGO will be able to detect gravitational signals emitted by the coalescence of a $1 M_{\odot}-1 M_{\odot}$ BWD up to $\sim 50 \mathrm{Mpc}$. Fixing the primary mass $m_{1}$ and following the line of constant $\rho=8$, we see that binary configurations with a mass ratio of $m_{1} / m_{2} \leq 1.5$ are luminous enough to be resolved by B-DECIGO. At $100 \mathrm{Mpc}$, DECIGO will observe all of the systems within the shaded area with a $\mathrm{S} / \mathrm{N}$ above the threshold. This will be crucial to explore the whole mass spectrum of the BWD coalescence, in detail, and its connection with $\mathrm{SN}$ events. We note that for the computation of the $\mathrm{S} / \mathrm{N}$, the distance acts as a scaling factor, namely $\rho \sim 1 / d$, and therefore our results can immediately be shifted to any value of $d$. A more detailed study, as a function of the source's localization, is described in Appendix B.

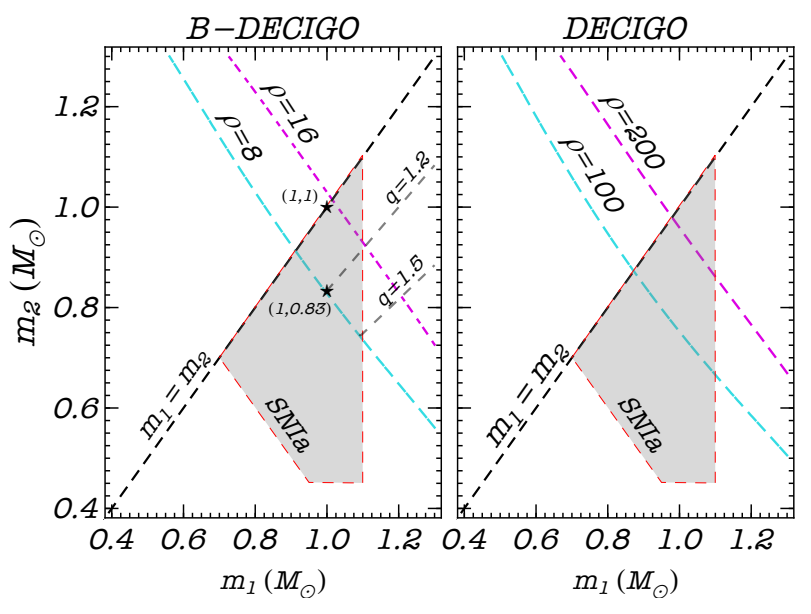

Fig. 2. Contour lines of fixed $\mathrm{S} / \mathrm{N} \rho$ as a function of the component masses $m_{1,2}$ for BWD observed by B-DECIGO (left panel) at $d=50$ $\mathrm{Mpc}$ and DECIGO (right panel) at $d=100 \mathrm{Mpc}$. The $\mathrm{S} / \mathrm{N}$ was computed by averaging over the source orientations. The shaded gray region identifies the region where the coalescence of the two stars may lead to supernovae Ia events (Postnov \& Yungelson 2014). We also show lines of constant mass ratio $q=m_{1} / m_{2}$.

The observations of $\mathrm{SNe}$ in the local Universe make it possible to derive volumetric rates for the different SN types as function of redshift. For the distances sampled by the present work, the volumetric rate of type Ia SNe is $0.25 \pm 0.05 \times 10^{-4} \mathrm{Mpc}^{-3} \mathrm{yr}^{-1}$ (Cappellaro et al. 2015, Li et al. 2011), which corresponds to $O(10) \mathrm{SNe}$ Type Ia events per year within a volume of $50 \mathrm{Mpc}$ radius and $O\left(10^{2}\right)$ for a volume of $100 \mathrm{Mpc}$ radius.

\subsection{Accuracy of the source parameters}

Since the parameter's space to sample is rather large, we focus on the following: (i) equal mass binaries with $m_{1}+m_{2}=2 M_{\odot}$; and (ii) specific values of the source distance and of the angular momentum's direction. For DECIGO (B-DECIGO), binaries are located $100 \mathrm{Mpc}(50 \mathrm{Mpc})$ from the detector. Although the mass distribution of BWD is currently not known, we expect that our result will not be dramatically affected by small variations of the mass ratio $q=m_{2} / m_{1} \cdot 3$

The Fisher approach allows one to derive bounds on all the source parameters; however, hereafter we only discuss uncertainties about the luminosity distance and on the angles that identify $\hat{L}$. In particular, in order to investigate the accuracy on $\bar{\theta}_{\mathrm{L}}$ and $\bar{\phi}_{\mathrm{L}}$ we introduce the error box on the solid angle spanned by unit vector $\hat{L}$ as (Cutler 1998):

$$
\Delta \Omega_{\mathrm{L}}=2 \pi\left|\sin \bar{\theta}_{\mathrm{L}}\right| \sqrt{\sigma_{\bar{\theta}_{\mathrm{L}}}^{2} \sigma_{\bar{\phi}_{\mathrm{L}}}^{2}-\Sigma_{\bar{\theta}_{\mathrm{L}} \bar{\phi}_{\mathrm{L}}}^{2}} .
$$

We first consider the case without any prior on the localization. In this framework we assume that no electromagnetic counterpart has been observed in coincidence with the gravitational wave event and, according to Sec. 2.1, we work with a full $9 \times 9$ Fisher matrix. This scenario is expected to be more frequen

The relative errors on the luminosity distance and on $\Delta \Omega_{\mathrm{L}}$ are shown in Table 1 for certain BWD's configurations, which feature $\mathrm{S} / \mathrm{Ns}$ in the range of $\rho^{\mathrm{DEC}} \in(131,290)$ and $\rho^{\mathrm{B}-\mathrm{DEC}} \in$ $(8,19)$. Independent of the particular combination of $\left(\bar{\theta}_{\mathrm{S}}, \bar{\phi}_{\mathrm{S}}\right)$ and $\left(\bar{\theta}_{\mathrm{L}}, \bar{\phi}_{\mathrm{L}}\right)$, the values of $\sigma_{d} / d$ obtained for DECIGO have a nearly flat distribution that clusters around $1 \%$. For the smaller interferometer, the uncertainties on $d$ have roughly the same spread. At $50 \mathrm{Mpc}$, we expect B-DECIGO to measure the luminosity distance of BWDs with $10 \%$ accuracy. Sources closer to the detector would improve these estimates. As an example, a system at $d=10 \mathrm{Mpc}$ with the same sky location of the second binary in Table 1 leads to $\sigma_{d}^{\mathrm{B}-\mathrm{DEC}} / d \simeq 1 \%$. It is also important to note that $d$ is almost uncorrelated with the other parameters, hence its error is proportional to the inverse of the $\mathrm{S} / \mathrm{N}$, namely $\sigma_{d} / d \sim 1 / \rho$, and our results can immediately be rescaled to any value of the luminosity distance.

We can now focus on the accuracy on the orientation of the binary's angular momentum. The last two columns of Table 2 show the projected constraints on $\Delta \Omega_{\mathrm{L}}$ defined in eq. (9). A satellite similar to DECIGO would estimate the direction of $\hat{L}$ with exquisite precision: For all of the models analyzed, we find a maximum error on $\Delta \Omega_{\mathrm{L}}$ smaller than one degree squared, even when considering sources at $100 \mathrm{Mpc}$ from the detector. For BDECIGO, the accuracy decreases of more than two orders of magnitude. At $d=50 \mathrm{Mpc}$, in the best case scenario, $\Omega_{\mathrm{L}}$ can be determined with roughly $67 \mathrm{deg}^{2}$ of accuracy.

\footnotetext{
3 As an example, for binary configurations with $q \gtrsim 1$, which is still compatible with the BWD-supernovae Ia scenario, the relative error of the luminosity distance $d$ would increase with the mass ratio, scaling at the leading order as $\sim \sqrt{q}$. We note, however, that for values of $q \gtrsim 1.2$, the merger may not be able to ignite the SN explosion (Pakmor et al. 2011).

${ }_{4}^{4}$ Possible absorption of the electromagnetic emission or large gravitational-wave localization may reduce the actual number of coincident detections (Rebassa-Mansergas et al. 2019).
} 

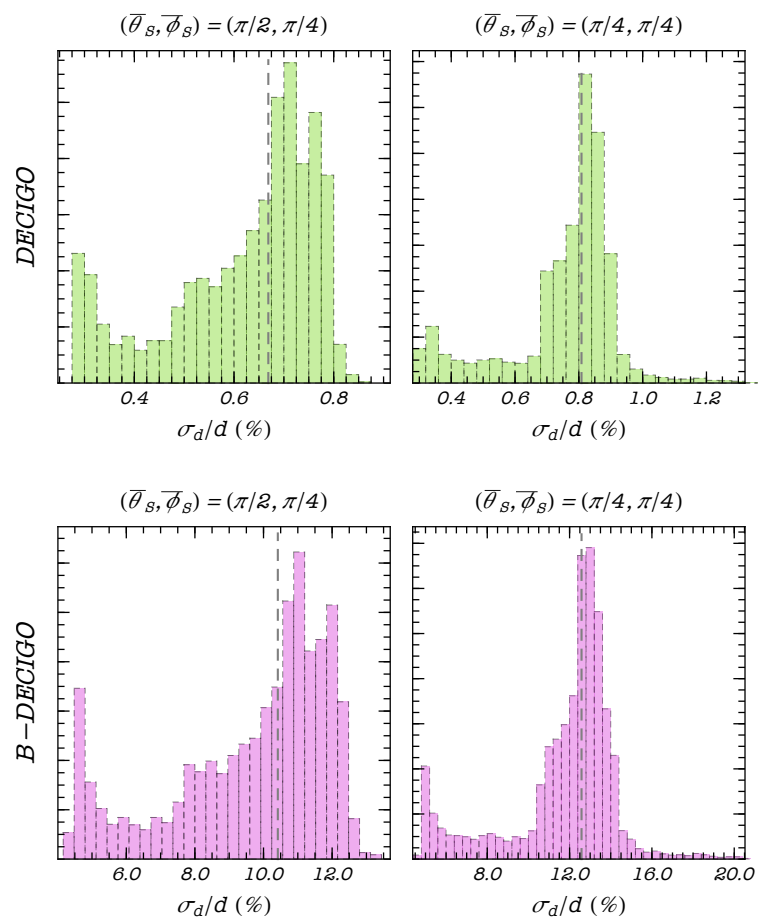

Fig. 3. 1- $\sigma$ distributions of errors for the luminosity distance inferred for $n=10^{4}$ sources with orbital angular momentum randomly distributed on the sphere and a fixed sky localization with respect to the interferometers configurations $\left(\bar{\theta}_{\mathrm{s}}, \bar{\phi}_{\mathrm{s}}\right)=(\pi / 2, \pi / 4)$ left plots and $\left(\bar{\theta}_{\mathrm{s}}, \bar{\phi}_{\mathrm{s}}\right)=(\pi / 4, \pi / 4)$ right plots. Vertical dashed lines identify the median of the distribution. The binary systems are located at $d=100 \mathrm{Mpc}$ and at $d=50 \mathrm{Mpc}$ for DECIGO and B-DECIGO observations, respectively. We consider equal mass WDs with $m_{1,2}=1 M_{\odot}$.

To further investigate the uncertainties on the luminosity distance and on the solid angle, we built an ensemble of $n=10^{4}$ BWDs in which $\cos \bar{\theta}_{\mathrm{L}}$ and $\bar{\phi}_{\mathrm{L}}$ are randomly drawn from uniform distributions within $[-1,1]$ and $[0,2 \pi]$, respectively. Masses and distances are assumed to be the same as before, while polar and azimuthal angles are fixed to specific values. For each system we computed the corresponding covariance matrix. Figures 3 . 5 show the histograms of the error's distributions on the luminosity distance for such configurations. The median of the 1$\sigma$ for DECIGO are $\sigma_{d} / d \simeq 0.67 \%$ and $\sigma_{d} / d \simeq 0.81 \%$ for $\bar{\theta}_{\mathrm{S}}=\pi / 2$ and $\bar{\theta}_{\mathrm{S}}=\pi / 4$, respectively. Here, we set $\bar{\phi}_{\mathrm{S}}=\pi / 4$, but the results are nearly identical for $\bar{\phi}_{\mathrm{S}}=0$. For B-DECIGO, such a median increases to $\sigma_{d} / d \simeq 10.4 \%$ and $\sigma_{d} / d \simeq 12.6 \%$ for the two cases considered, namely $\left(\bar{\theta}_{\mathrm{S}}, \bar{\phi}_{\mathrm{S}}\right)=(\pi / 2, \pi / 4)$ and $\left(\bar{\theta}_{\mathrm{S}}, \bar{\phi}_{\mathrm{S}}\right)=(\pi / 4, \pi / 4)$. These values are in agreement with the single-source analysis shown in Table 1 . Finally, the two panels of Fig. 6 show the cumulative density function of $\Delta \Omega_{\mathrm{L}}$ for the same set of $n=10^{4}$ binaries. For DECIGO, $90 \%$ of the population yields $\Delta \Omega_{\mathrm{L}} \lesssim 1 \mathrm{deg}^{2}$ for $\bar{\theta}_{\mathrm{S}}=\pi / 2$, with the results being very similar for the case with $\bar{\theta}_{\mathrm{S}}=\pi / 4$. The analysis for B-DECIGO leads to larger uncertainties, and the almost totality of the configurations sampled yield errors of $\Delta \Omega_{\mathrm{L}} \gtrsim 100$ $\mathrm{deg}^{2}$. We note that the knowledge of the angular momentum's direction represents a crucial piece of information that can be inferred from the binary's orbital motion. Assuming that the masses are also determined by the GW analysis, the measurement of $\hat{L}$ would help to reconstruct the full morphology of the system. For the multimessenger detections discussed below, this implies characterizing the BWD's evolution from the inspiral to the supernovae event.
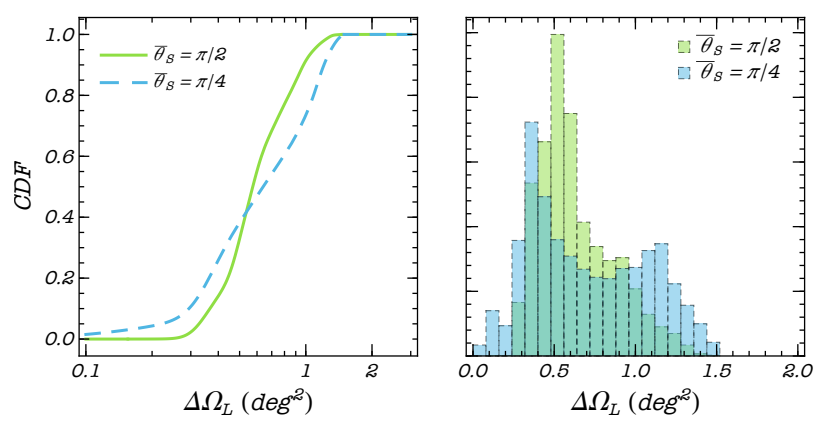

Fig. 4. Cumulative (left panel) and histogram (right panel) distribution of the 1- $\sigma$ errors on the solid angle $\Omega_{\mathrm{L}}$ derived from the ensemble of $n=$ $10^{4}$ binary white dwarf with angular momentum randomly oriented and specific configurations of $\left(\bar{\theta}_{\mathrm{S}}, \bar{\phi}_{\mathrm{S}}\right)$. Sources are observed by DECIGO at $d=100 \mathrm{Mpc}$.

\subsection{Multimessenger detections and the Hubble constant}

We now focus on the multimessenger scenario in which a SN Ia event is observed in coincidence with the GW signal. In this case, we assume that the binary's polar and azimuthal angles are known. In practice, we removed $\bar{\theta}_{\mathrm{S}}$ and $\bar{\phi}_{\mathrm{S}}$ from the Fisher matrix, reducing the number of parameters to constrain. The values of $\sigma_{d} / d$ and $\Delta \Omega_{\mathrm{L}}$ that were computed following this approach are shown between round brackets in Table 1 For the angles chosen in Table 1. the uncertainties of both the luminosity distance and the solid angle are close to those derived in the previous section. For both DECIGO and B-DECIGO, we obtain differences up to $4 \%$ on $d$ and $6 \%$ on $\Delta \Omega_{\mathrm{L}}$ between the $1-\sigma$ derived with and without the supernova prior. We have repeated this analysis for the statistical ensemble of $10^{4}$ binaries shown in Figs. (3)(5), and we find, however, that some particular combinations of the source orientation may lead to discrepancies on the order of $10 \%$ on the luminosity distance and up to $100 \%$ on the angular momentum direction. Multimessenger constraints therefore harbor the potential to significantly increase the accuracy of the parameter estimation.

However, the observation of the electromagnetic counterpart is also crucial in disentangling the source's redshift. The latter can be used to translate constraints of the luminosity distance into bounds on the Hubble constant. We show such values in Table 2. The errors of $H_{0}$ that were computed for DECIGO are smaller than the local measurements inferred using supernovae observations (Riess et al.2019) or gravitational lensing time delays (Wong et al. 2019), and they are comparable with those obtained from the cosmic microwave background Aghanim et al. 2018) (see also Fig. 1). We also note that the exquisite precision of DECIGO makes it so $\sigma_{H_{0}}$ is dominated by the uncertainty as to the peculiar velocity which, at $100 \mathrm{Mpc}$, can be up to a factor $\sim 2$ higher than the errors coming from the luminosity distance alone. As expected, uncertainties for B-DECIGO are, in general, larger. At $50 \mathrm{Mpc}$, the projected constraints are looser than current bounds in the electromagnetic band, although they are still smaller than the value derived for the first double neutron star GW event (Abbott et al. 2017a). The last two columns of Table 2 also show that for B-DECIGO, the uncertainty of the Hub- 


\begin{tabular}{rccccccc}
\hline \hline $\cos \bar{\theta}_{\mathrm{L}}$ & $\bar{\phi}_{\mathrm{L}}$ & $\cos \bar{\theta}_{\mathrm{S}}$ & $\bar{\phi}_{\mathrm{S}}$ & $\sigma_{d}^{\mathrm{DEC}} / d \%$ & $\sigma_{d}^{\mathrm{B}-\mathrm{DEC}} / d \%$ & $\Delta \Omega_{\mathrm{L}}^{\mathrm{DEC}}$ & $\Delta \Omega_{\mathrm{L}}^{\mathrm{B}-\mathrm{DEC}}$ \\
\hline-0.2 & 4 & 0.3 & 5 & $0.787(0.806)$ & $12.3(12.6)$ & $0.899(0.942)$ & $219(229)$ \\
0.2 & 0 & 0.3 & 5 & $0.561(0.562)$ & $8.75(8.76)$ & $0.284(0.298)$ & $69.2(72.6)$ \\
-0.2 & 4 & -0.3 & 1 & $0.391(0.391)$ & $6.10(6.10)$ & $0.379(0.380)$ & $92.4(92.6)$ \\
0.2 & 0 & -0.3 & 1 & $0.777(0.796)$ & $12.1(12.4)$ & $0.895(0.938)$ & $218(228)$ \\
-0.2 & 4 & 0.3 & 3 & $0.563(0.564)$ & $8.74(8.76)$ & $0.289(0.303)$ & $70.0(73.5)$ \\
0.2 & 0 & 0.3 & 3 & $0.780(0.812)$ & $12.1(12.7)$ & $0.905(0.958)$ & $219(232)$ \\
-0.2 & 4 & -0.3 & 6 & $0.542(0.542)$ & $8.41(8.41)$ & $(0.278)(0.294)$ & $67.4(71.2)$ \\
0.2 & 0 & -0.3 & 6 & $0.768(0.779)$ & $11.9(12.1)$ & $0.838(0.872)$ & $202(211)$ \\
-0.8 & 4 & 0.5 & 5 & $0.774(0.774)$ & $12.1(12.1)$ & $0.35(0.353)$ & $85.0(85.9)$ \\
0.8 & 0 & 0.5 & 5 & $0.806(0.816)$ & $12.6(12.7)$ & $0.466(0.486)$ & $114(119)$ \\
-0.8 & 4 & -0.5 & 1 & $0.741(0.742)$ & $11.6(11.6)$ & $0.408(0.429)$ & $98.7(104)$ \\
0.8 & 0 & -0.5 & 1 & $0.762(0.762)$ & $11.9(11.9)$ & $0.357(0.360)$ & $86.9(87.9)$ \\
-0.8 & 4 & 0.5 & 3 & $0.797(0.805)$ & $12.4(12.5)$ & $0.409(0.424)$ & $98.8(102)$ \\
0.8 & 0 & 0.5 & 3 & $0.749(0.749)$ & $11.6(11.7)$ & $0.352(0.358)$ & $84.8(86.4)$ \\
-0.8 & 4 & -0.5 & 6 & $0.783(0.792)$ & $12.2(12.3)$ & $0.49(0.508)$ & $118(123)$ \\
0.8 & 0 & -0.5 & 6 & $0.760(0.760)$ & $11.8(11.8)$ & $0.323(0.325)$ & $77.9(78.4)$ \\
\hline
\end{tabular}

Table 1. $1-\sigma$ uncertainties of the luminosity distance $d$ (relative percentual) and of the solid angle (deg ${ }^{2}$ ) identified by the binary angular momentum for certain combinations of $\left(\bar{\theta}_{\mathrm{S}}, \bar{\phi}_{\mathrm{S}}\right)$ and of the angles $\left(\bar{\theta}_{\mathrm{L}}, \bar{\phi}_{\mathrm{L}}\right)$. White dwarf binaries have masses $m_{1,2}=1 M_{\odot}$, and are placed at $d=100 \mathrm{Mpc}$ and $d=50 \mathrm{Mpc}$, from DECIGO and B-DECIGO, respectively. Values between round brackets correspond to errors evaluated for the same configuration through the multimessenger analysis, i.e. knowing the source localization given by $\left(\bar{\theta}_{\mathrm{S}}, \bar{\phi}_{\mathrm{S}}\right)$.

ble parameter is dominated by the statistical error coming from the GW parameter estimation.
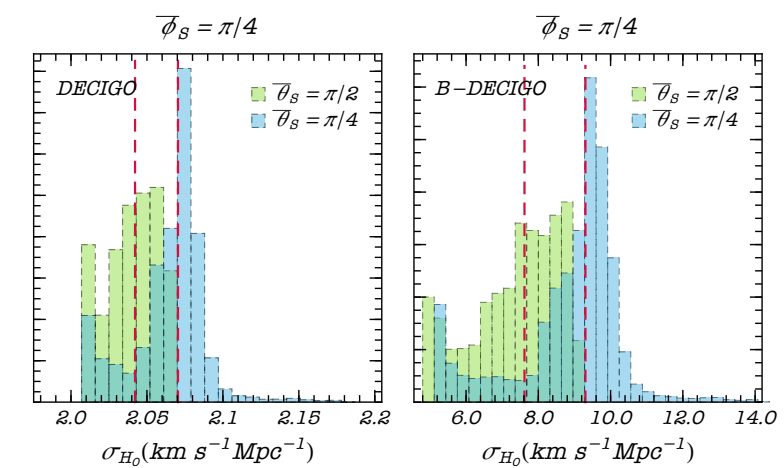

Fig. 5. Error distribution of $1-\sigma$ for the Hubble constant computed for the set of $n=10^{4}$ binary white dwarfs also considered in Fig. 3 .

The two panels of Fig. 5 show the errors on $H_{0}$ that were computed for the random set of $10^{4}$ binaries introduced in the previous section. Sources with smaller values of $\bar{\theta}_{\mathrm{S}}$ lead to larger uncertainties for both the detectors. This picture does not change dramatically if we vary $\bar{\phi}_{\mathrm{S}}$. Overall, we find medians, which were corrected by the peculiar velocity, for DECIGO of $\sigma_{H_{0}} \simeq 2.04$ $\mathrm{km} \mathrm{s}^{-1} \mathrm{Mpc}^{-1}\left(\bar{\theta}_{\mathrm{S}}=\pi / 2\right)$ and $\sigma_{H_{0}} \simeq 2.07 \mathrm{~km} \mathrm{~s}^{-1} \mathrm{Mpc}^{-1}\left(\bar{\theta}_{\mathrm{S}}=\right.$ $\pi / 4)$. For B-DECIGO, such values increase to $\sigma_{H_{0}} \simeq 7.62 \mathrm{~km}$ $\mathrm{s}^{-1} \mathrm{Mpc}^{-1}$ and $\sigma_{H_{0}} \simeq 9.30 \mathrm{~km} \mathrm{~s}^{-1} \mathrm{Mpc}^{-1}$. Therefore, in complete analogy with the coalescence of neutron stars and their gammaray-burst counterpart (Abbott et al.2017a), multimessenger observations of BWDs provide a powerful tool to determine the local value of the Hubble constant, which is independent and competitive with current constraints.

In addition to the cosmological application, coincident detections of merging WDs may also be used to calibrate SN Ia luminosities, as was recently proposed in (Gupta et al. 2019) by comparing the gravitational and the electromagnetic measurements of $d$. In this work, the authors consider GWs that were

\begin{tabular}{rrrrrrrrr}
\hline \hline $\cos \bar{\theta}_{\mathrm{L}}$ & $\bar{\phi}_{\mathrm{L}}$ & $\cos \bar{\theta}_{\mathrm{S}}$ & $\bar{\phi}_{\mathrm{S}}$ & $\sigma_{H_{0}}^{\mathrm{DEC}}$ & $\sigma_{H_{0}, v e l}^{\mathrm{DEC}}$ & $\sigma_{H_{0}}^{\mathrm{B}-\mathrm{DEC}}$ & $\sigma_{H_{0}, v e l}^{\mathrm{B}-\mathrm{DEC}}$ \\
\hline-0.2 & 4 & 0.3 & 5 & 0.522 & 2.07 & 8.21 & 9.13 \\
0.2 & 0 & 0.3 & 5 & 0.372 & 2.03 & 5.85 & 7.09 \\
-0.2 & 4 & -0.3 & 1 & 0.259 & 2.02 & 4.08 & 5.71 \\
0.2 & 0 & -0.3 & 1 & 0.515 & 2.07 & 8.08 & 9.02 \\
-0.2 & 4 & 0.3 & 3 & 0.373 & 2.03 & 5.84 & 7.08 \\
0.2 & 0 & 0.3 & 3 & 0.517 & 2.07 & 8.11 & 9.05 \\
-0.2 & 4 & -0.3 & 6 & 0.359 & 2.03 & 5.62 & 6.90 \\
0.2 & 0 & -0.3 & 6 & 0.509 & 2.06 & 7.98 & 8.93 \\
-0.8 & 4 & 0.5 & 5 & 0.513 & 2.06 & 8.08 & 9.02 \\
0.8 & 0 & 0.5 & 5 & 0.534 & 2.07 & 8.42 & 9.32 \\
-0.8 & 4 & -0.5 & 1 & 0.492 & 2.06 & 7.75 & 8.72 \\
0.8 & 0 & -0.5 & 1 & 0.505 & 2.06 & 7.94 & 8.89 \\
-0.8 & 4 & 0.5 & 3 & 0.528 & 2.07 & 8.29 & 9.20 \\
0.8 & 0 & 0.5 & 3 & 0.496 & 2.06 & 7.78 & 8.75 \\
-0.8 & 4 & -0.5 & 6 & 0.519 & 2.07 & 8.14 & 9.07 \\
0.8 & 0 & -0.5 & 6 & 0.504 & 2.06 & 7.90 & 8.85 \\
\hline
\end{tabular}

Table $\overline{\overline{2 \text {. Uncertainties of the Hubble constant } H_{0} \text { (in } \mathrm{km} \mathrm{s}^{-1}}} \mathrm{Mpc}^{-1}$ ) evaluated by coincident electromagnetic and gravitational wave detections of the same binary configurations shown in Table 1 . For each configuration, we show the error corrected with and without the velocity dispersion.

emitted by neutron star mergers, occurring in galaxies that host supernovae explosions. This strategy, however, may suffer from spurious systematics, as there is a lack of precise knowledge on the relative position between the two events. In our approach, the association between the WDs merger and the SN Ia, through the double degenerate scenario, would provide a unique and consistent estimate of the source's luminosity distances and of its flux.

\section{Conclusions}

Along with black holes and neutron stars, white dwarfs represent one of the flavors in which compact objects manifest in 
the Universe. Similar to single or binary sources, their evolution leads to a rich phenomenology, which is connected with a large variety of astrophysical phenomena (Postnov \& Yungelson 2014; Shen 2015). Depending on the nature of the companion, WD coalescences could ignite the emission of different electromagnetic counterparts, as X-transients (Sesana et al. 2008; Bauer et al. 2017). Moreover, binary WDs have received a lot of attention as possible progenitors of supernovae Ia events, according to the double degenerate scenario. For these reasons, they represent ideal candidates to fully exploit multimessenger observations.

In this paper, we investigate the detectability of coalescing white dwarf systems at the end of their inspiral phase via decihertz gravitational wave interferometers. We computed the $\mathrm{S} / \mathrm{N}$ for prototype binaries, assessing the accuracy of the source's parameters estimated by the Japanese detectors DECIGO and B-DECIGO. We primarily focus on the constraints that can be placed on the luminosity distance. Indeed, since the orbital evolution of a compact binary is completely determined by general relativity, BWDs are clean standard sirens. We find that DECIGO can measure the source's distance with $1 \%$ accuracy and with better accuracy for binaries at $100 \mathrm{Mpc}$ from the detector. $\mathrm{B}$-DECIGO is able to perform the same quality-measurements for systems one order of magnitude closer, that is, for $d<20$ Mpc. Although, the interferometer will still constrain the luminosity distance with a relative accuracy of $10 \%$ within an horizon of $50 \mathrm{Mpc}$.

We explore the multimessenger scenario, in which GWs signals are observed in coincidence with SN Ia events, with the latter providing the source's polar and azimuthal angles. Overall, we find a mild improvement of the statistical errors on $d$. However, the joint analysis has a crucial impact on cosmology: Assuming that the electromagnetic channel only yields the binary's redshift, the local value of the Hubble constant can now be determined. Our results suggest that DECIGO (B-DECIGO) can put a bound on $H_{0}$, such that at a $68 \%$ confidence level, $\sigma_{H_{0}} \lesssim 2.1$ $\left(\sigma_{H_{0}} \lesssim 5.7\right) \mathrm{km} \mathrm{s}^{-1} \mathrm{Mpc}^{-1}$ at $d=100 \mathrm{Mpc}(d=50 \mathrm{Mpc})$. The strategy devised in this paper is complementary to measurements at low redshift that are available nowadays and, therefore, it offers an independent approach to alleviate or solve the tension on the Hubble constant.

While for binary neutron stars, the beamed electromagnetic emission can constrain the orbital plane and thus improve the distance and the Hubble constant measurements (Hotokezaka et al. 2019, Chen et al. 2019); for BWD, the $H_{0}$ measurement precision benefits from a higher rate of joint detections, due to the isotropic emission of SN type Ia with respect to the GRB and a brighter emission with respect to a kilonova, and from a higher astrophysical rate of BWD with respect to the BNS rate (the white-dwarf merger rate is about 1-2 orders of magnitude higher than for BNS) (Badenes \& Maoz 2012, Abbott et al. 2017g). Indeed, the projected constraints discussed so far can be further improved by stacking multiple GW observations. To show how $\sigma_{H_{0}}$ changes, we simulated one thousand sets of $100 \mathrm{GW}$ detections each one. For each binary the primary mass and mass ratio are drawn randomly from uniform distributions within the intervals $m_{1} \in[0.8,1.1] M_{\odot}$ and $q=m_{2} / m_{1} \in[1,1.2]$. The source orientations and angular momenta were also randomly chosen on the sphere. The luminosity distance of such events is picked from a uniform distribution between 50 and $200 \mathrm{Mpc}$. We stacked a different number of observations, which shows the corresponding errors for DECIGO in Fig. 6. By comparing such values with the uncertainties found in Fig. 3 and Table 2, we note that exploiting the white-dwarf merger rate would reduce the error on the Hubble constant by almost one order of magnitude with 100 detections.

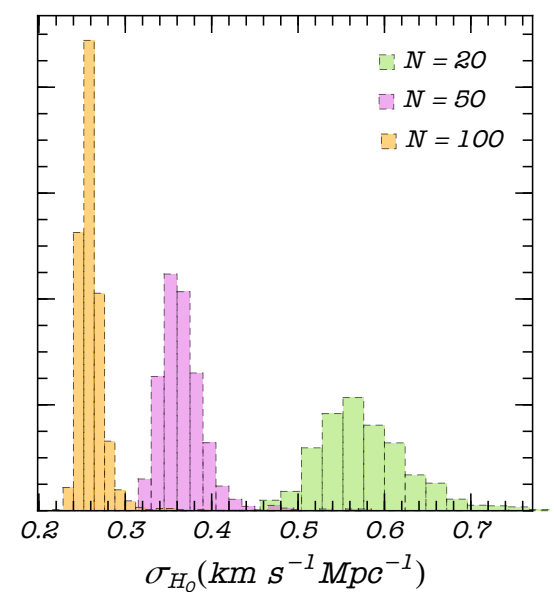

Fig. 6. Projected 1- $\sigma$ constraints on the value of the Hubble constant measured with DECIGO as a function of the number of GW observations.

In addition to the cosmological implications, coincident detections of binary white dwarfs may be used to calibrate the supernova's light-curves, as recently suggested in Gupta et al. (2019). We remark that confirmation of the double degenerate scenario would provide a fully consistent calibration of the SN flux, free of any systematics due to missing information between the electromagnetic and the gravitational event, which is in analogy with GW170817 (Abbott et al.2017a).

This statistical study also allowed us to determine the accuracy with which decihertz interferometers will be able to measure the inclination of the BWD's orbital plane. We find that DECIGO can constrain the solid angle identified by the orbital angular momentum with accuracies below $3 \mathrm{deg}^{2}$. This represents an important piece of information, which characterizes the binary's evolution prior the merger, and therefore it may have deep implications for the supernova explosion.

The data analysis carried out in this paper does not take finite size effects due to rotation or tidal interactions into account (Piro 2011, McNeill et al. 2019, Fuller \& Lai 2012). Such corrections affect the waveform at higher post-Newtonian order, and they are expected to be small compared to the dominant contribution (Willems et al. 2010; Valsecchi et al. 2012). However, we plan to improve upon the GW description and further explore the impact these modifications have in a forthcoming publication. As a final remark, due to the relevance of binary white dwarf within the stellar evolution, it would be interesting to study the source localization of such systems in the millihertz band spanned by LISA. A detailed study on this topic, which also exploits a catalog of binaries sampled and adopts the approach described in Schneider et al. (2017), Graziani et al. (2017) and Marassi et al. (2019), is already ongoing (Maselli \& others. 2020).

Acknowledgements. A.M wants to thank Swetha Bhagwat and Luca Izzo for useful discussions and for having carefully read this manuscript. The Authors also thank the organizers of the PAX meeting, "Physics and Astrophysics at the eXtreme", held in Cascina in 2019, where the idea of this work was discussed. A.M acknowledges support from the Amaldi Research Center funded by the MIUR program "Dipartimento di Eccellenza" (CUP: B81I18001170001). The authors would like to acknowledge networking support by the COST Action CA16104. 


\section{References}

Abbott, B. P. et al. 2016a, Phys. Rev. Lett., 116, 241103 Abbott, B. P. et al. 2016b, Phys. Rev. Lett., 116, 061102 Abbott, B. P. et al. 2017a, Nature, 551, 85

Abbott, B. P. et al. 2017b, Class. Quant. Grav., 34, 044001

Abbott, B. P. et al. 2017c, Astrophys. J., 848, L13

Abbott, B. P. et al. 2017d, Phys. Rev. Lett., 118, 221101, [Erratum: Phys. Rev. Lett.121,no.12,129901(2018)]

Abbott, B. P. et al. 2017e, Phys. Rev. Lett., 119, 141101

Abbott, B. P. et al. 2017f, Phys. Rev. Lett., 119, 161101

Abbott, B. P. et al. $2017 \mathrm{~g}$, Astrophys. J., 850, L40

Abbott, B. P. et al. 2018 [arXiv: 1811.12907]

Addison, G. E., Watts, D. J., Bennett, C. L., et al. 2018, Astrophys. J., 853, 119

Aghanim, N. et al. 2018 [arXiv: 1807.06209]

Akutsu, T. et al. 2019, Nat. Astron., 3, 35

Apostolatos, T. A., Cutler, C., Sussman, G. J., \& Thorne, K. S. 1994, Phys. Rev., $\mathrm{D} 49,6274$

Audley, H. et al. 2017 [arXiv: 1702.00786]

Aylor, K., Joy, M., Knox, L., et al. 2019, Astrophys. J., 874, 4

Badenes, C. \& Maoz, D. 2012, Astrophys. J., 749, L11

Barack, L. et al. 2019, Class. Quant. Grav., 36, 143001

Bauer, F. E. et al. 2017, Mon. Not. Roy. Astron. Soc., 467, 4841

Berti, E., Buonanno, A., \& Will, C. M. 2005, Phys. Rev., D71, 084025

Cappellaro, E. et al. 2015, Astron. Astrophys., 584, A62

Carrick, J., Turnbull, S. J., Lavaux, G., \& Hudson, M. J. 2015, Mon. Not. Roy. Astron. Soc., 450, 317

Carson, Z. \& Yagi, K. 2019 [arXiv: 1905.13155]

Chen, H.-Y., Fishbach, M., \& Holz, D. E. 2018, Nature, 562, 545

Chen, H.-Y., Vitale, S., \& Narayan, R. 2019, Physical Review X, 9, 031028

Cutler, C. 1998, Phys. Rev., D57, 7089

Dalal, N., Holz, D. E., Hughes, S. A., \& Jain, B. 2006, Phys. Rev., D74, 063006

Damour, T., Nagar, A., \& Villain, L. 2012, Phys. Rev., D85, 123007

Dwyer, S., Sigg, D., Ballmer, S. W., et al. 2015, Phys. Rev., D91, 082001

Feeney, S. M., Peiris, H. V., Williamson, A. R., et al. 2019, Phys. Rev. Lett., 122, 061105

Finn, L. S. \& Chernoff, D. F. 1993, Phys. Rev., D47, 2198

Fuller, J. \& Lai, D. 2012, Mon. Not. Roy. Astron. Soc., 420, 3126

Gnocchi, G., Maselli, A., Abdelsalhin, T., Giacobbo, N., \& Mapelli, M. 2019 [arXiv: 1905.13460]

Graziani, L., de Bennassuti, M., Schneider, R., Kawata, D., \& Salvadori, S. 2017, Mon. Not. Roy. Astron. Soc., 469, 1101

Gupta, A., Fox, D., Sathyaprakash, B. S., \& Schutz, B. F. 2019 [arXiv: 1907.09897]

Hamuy, M., Phillips, M. M., Suntzeff, N. B., et al. 1996, Astron. J., 112, 2398

Holz, D. E. \& Hughes, S. A. 2005, Astrophys. J., 629, 15

Hotokezaka, K., Nakar, E., Gottlieb, O., et al. 2019, Nature Astronomy, 385

Iben, Jr., I. \& Tutukov, A. V. 1984, "Astrophys. J. Suppl. Series", 54, 335

Isoyama, S., Nakano, H., \& Nakamura, T. 2018, PTEP, 2018, 073E01

Kawamura, S. et al. 2011, Class. Quant. Grav., 28, 094011

Khan, S., Husa, S., Hannam, M., et al. 2016, Phys. Rev., D93, 044007

Kim, A. G. et al. 1997, Astrophys. J., 476, L63

Kowalska, I., Bulik, T., \& Belczynski, K. 2012, Astron. Astrophys., 541, A120

Krolak, A. \& Schutz, B. F. 1987, General Relativity and Gravitation, 19, 1163

Li, W., Chornock, R., Leaman, J., et al. 2011, Mon. Not. Roy. Astron. Soc., 412, 1473

Littenberg, T. B., Breivik, K., Brown, W. R., et al. 2019 [arXiv: 1903.05583]

Macaulay, E. et al. 2019, Mon. Not. Roy. Astron. Soc., 486, 2184

Mandel, I., Sesana, A., \& Vecchio, A. 2018, Class. Quant. Grav., 35, 054004

Maoz, D., Mannucci, F., \& Nelemans, G. 2014, Ann. Rev. Astron. Astrophys., 52,107

Marassi, S., Graziani, L., Ginolfi, M., et al. 2019, Mon. Not. Roy. Astron. Soc., 484, 3219

Marassi, S., Schneider, R., \& Ferrari, V. 2009, Mon. Not. Roy. Astron. Soc., 398, 293

Marković, D. 1993, Phys. Rev. D, 48, 4738

Maselli, A. \& others. 2020, To be submitted

McNeill, L. O., Mardling, R. A., \& Müller, B. 2019 [arXiv: 1901.09045]

McWilliams, S. T., Caldwell, R., Holley-Bockelmann, K., Larson, S. L., \& Vallisneri, M. 2019 [arXiv: 1903.04592]

Nair, R. \& Tanaka, T. 2018, JCAP, 1808, 033, [Erratum: JCAP1811,no.11,E01(2018)]

Nauenberg, M. 1972, ApJ, 175, 417

Nelemans, G., Yungelson, L. R., \& Portegies Zwart, S. F. 2001, Astron. Astrophys., 375,890

Nielsen, M. T. B., Nelemans, G., Voss, R., \& Toonen, S. 2014, Astron. Astrophys., 563 , A16

Nissanke, S., Holz, D. E., Hughes, S. A., Dalal, N., \& Sievers, J. L. 2010, Astrophys. J., 725, 496
Pakmor, R., Hachinger, S., Röpke, F. K., \& Hillebrand t, W. 2011, "Astron. Astrophys.", 528, A117

Piro, A. L. 2011, Astrophys. J., 740, L53

Poisson, E. \& Will, C. M. 1995, Physical Review D, 52, 848

Portegies Zwart, S. F. \& Verbunt, F. 1996, A\&A, 309, 179

Postnov, K. A. \& Yungelson, L. R. 2014, Living Rev. Rel., 17, 3

Punturo, M. et al. 2010, Class. Quant. Grav., 27, 194002

Raskin, C., Scannapieco, E., Fryer, C., Rockefeller, G., \& Timmes, F. X. 2012, Astrophys. J., 746, 62

Rebassa-Mansergas, A., Toonen, S., Korol, V., \& Torres, S. 2019, Monthly Notices of the Royal Astronomical Society, 482, 3656

Riess, A. G., Casertano, S., Yuan, W., Macri, L. M., \& Scolnic, D. 2019, Astrophys. J., 876,85

Riess, A. G. et al. 1998, Astron. J., 116, 1009

Sathyaprakash, B. S. et al. 2019 [arXiv: 1903.09221]

Sato, S. et al. 2017, J. Phys. Conf. Ser., 840, 012010

Schneider, R., Ferrari, V., Matarrese, S., \& Portegies Zwart, S. F. 2001, Mon. Not. Roy. Astron. Soc., 324, 797

Schneider, R., Graziani, L., Marassi, S., et al. 2017, Mon. Not. Roy. Astron. Soc., 471, L105

Schutz, B. F. 1986, Nature, 323, 310

Sedda, M. A. et al. 2019 [arXiv: 1908.11375]

Sesana, A., Vecchio, A., Eracleous, M., \& Sigurdsson, S. 2008, Mon. Not. Roy. Astron. Soc., 391, 718

Shen, K. J. 2015, Astrophys. J.1, 805, L6

Tutukov, A. V. \& Yungelson, L. R. 1981, Nauchnye Informatsii, 49, 3

Usman, S. A., Mills, J. C., \& Fairhurst, S. 2019, Astrophys. J., 877, 82

Vallisneri, M. 2008, Physical Review D, 77, 042001

Valsecchi, F., Farr, W. M., Willems, B., Deloye, C. J., \& Kalogera, V. 2012, Astrophys. J., 745, 137

Vines, J., Flanagan, E. E., \& Hinderer, T. 2011, Phys. Rev., D83, 084051

Wang, B. 2018, Research in Astronomy and Astrophysics, 18, 049

Wang, B. \& Han, Z. 2012, New Astron. Rev., 56, 122

Webbink, R. F. 1984, "Astrophys. J.", 277, 355

Whelan, J. \& Iben, Jr., I. 1973, "Astrophys. J.", 186, 1007

Willems, B., Deloye, C. J., \& Kalogera, V. 2010, Astrophys. J., 713, 239

Wong, K. C. et al. 2019 [arXiv: 1907.04869]

Yagi, K. 2012, Class. Quant. Grav., 29, 075005

Yagi, K. \& Seto, N. 2011, Phys. Rev., D83, 044011, [Erratum: Phys. Rev.D95,no.10,109901(2017)]

Article number, page 8 of 10 


\section{Appendix A: Detector configuration}

In this section we give a brief review of the parameters that characterize the orbital configuration of DECIGO and B-DECIGO. We refer the reader to Cutler (1998) for a detailed analysis of the source's localization by space interferometers with an instrumental design similar to the one considered in this paper.

DECIGO is planned to be composed of four clusters of three spacecrafts each. The latter are separated by $1000 \mathrm{~km}$, forming a triangular configuration that moves on a Helio-centric orbit (Sato et al. 2017). In this configuration, we identified two reference systems: one aligned with the detector, and one fixed with the barycenter. We attached the coordinates $(x, y, z)$ and $(\bar{x}, \bar{y}, \bar{z})$ to these two systems, respectively. The arms of the interferometer lie in the $x-y$ plane, while the $z$-axis is inclined at an angle of $\gamma=\pi / 3$ with respect to $\bar{z}$ and it precesses around the latter at a constant rate, such that:

$\left.z^{i}=\frac{\bar{z}^{i}}{2}-\frac{\sqrt{3}}{2}[\cos \phi \overline{(} t) \bar{x}^{j}+\sin \phi \overline{(t)} \bar{y}^{j}\right]$

where $\left(x^{j}, y^{j}, z^{j}\right)$ are the unit vectors along the $(z, y, z)$-axis (and similarly for the barred coordinate). The orbital motion of the satellite is specified by $\bar{\phi}(t)=\phi_{0}+2 \pi t / T$ and $\bar{\theta}(t)=\pi / 2$, with $T=1$ year, and $\phi_{0}=0$ specifying the initial orientation with respect to the fixed reference frame. In projecting eq. A.1 on the unit vector that identifies the binary, we obtain the source's polar angle in the detector moving frame as a function of the fixed sky position:

$\cos \theta_{\mathrm{S}}=\frac{1}{2} \cos \bar{\theta}_{\mathrm{S}}-\frac{\sqrt{3}}{2} \sin \bar{\theta}_{\mathrm{S}} \cos \left[\bar{\phi}(t)-\bar{\phi}_{\mathrm{S}}\right]$,

and similarly for the azimuth:

$\bar{\phi}_{\mathrm{S}}=\tan ^{-1}\left[\frac{\sqrt{3} \cos \bar{\theta}_{\mathrm{S}}+\sin \bar{\theta}_{\mathrm{S}} \cos \left[\bar{\phi}(t)-\bar{\phi}_{\mathrm{S}}\right]}{2 \sin \bar{\theta}_{\mathrm{S}} \sin \left[\bar{\phi}(t)-\bar{\phi}_{\mathrm{S}}\right]}\right]$.

The polarization angle $\psi_{\mathrm{S}}$ also changes with time:

$\psi_{\mathrm{S}}(t)=\tan ^{-1} \frac{\hat{L} \cdot \hat{z}-(\hat{L} \cdot \hat{N})(\hat{z} \cdot \hat{N})}{\hat{N} \cdot(\hat{L} \times \hat{z})}$,

with $\hat{z} \cdot \hat{N}=\cos \theta_{\mathrm{S}}$ and

$$
\begin{aligned}
\hat{L} \cdot \hat{z} & =\frac{1}{2} \cos \bar{\theta}_{\mathrm{L}}-\frac{\sqrt{3}}{2} \sin \bar{\theta}_{\mathrm{L}} \cos \left[\bar{\phi}(t)-\bar{\phi}_{\mathrm{L}}\right], \\
\hat{L} \cdot \hat{N} & =\cos \bar{\theta}_{\mathrm{L}} \cos \bar{\theta}_{\mathrm{S}}+\sin \bar{\theta}_{\mathrm{L}} \sin \bar{\theta}_{\mathrm{S}} \cos \left[\bar{\phi}_{\mathrm{L}}-\bar{\phi}_{\mathrm{S}}\right],
\end{aligned}
$$

$\hat{N} \cdot(\hat{L} \times \hat{z})=\frac{1}{2} \sin \bar{\theta}_{\mathrm{L}} \sin \bar{\theta}_{\mathrm{S}} \sin \left[\bar{\phi}_{\mathrm{L}}-\bar{\phi}_{\mathrm{S}}\right]+$

$$
\begin{aligned}
& \frac{\sqrt{3}}{2}\left\{\cos \bar{\theta}_{\mathrm{L}} \sin \bar{\theta}_{\mathrm{L}} \sin \left[\bar{\phi}(t)-\bar{\phi}_{\mathrm{S}}\right]\right. \\
& \left.-\cos \bar{\theta}_{\mathrm{S}} \sin \bar{\theta}_{\mathrm{L}} \sin \left[\bar{\phi}(t)-\bar{\phi}_{\mathrm{L}}\right]\right\} .
\end{aligned}
$$

Finally, the Doppler phase shift defined in eq. (1) can be written as $\psi_{\mathrm{D}}=2 \pi f R_{\mathrm{AU}} \sin \bar{\theta}_{\mathrm{S}} \cos \left[\bar{\phi}(t)-\bar{\phi}_{\mathrm{S}}\right]$, where $R_{\mathrm{AU}}$ is the astronomical unit. For DECIGO, we assume that the noise spectral density is given by the following expression:

$$
\begin{aligned}
\frac{S_{h}^{\mathrm{D}}(f)}{\mathrm{Hz}^{-1}}=7.05 \cdot 10^{-48} y & +\frac{4.8 \cdot 10^{-51}}{y}\left(\frac{f}{\mathrm{~Hz}}\right)^{-4} \\
& +5.33 \cdot 10^{-52}\left(\frac{f}{\mathrm{~Hz}}\right)^{-4},
\end{aligned}
$$

with $y=1+\left(\frac{f}{7.36 \mathrm{~Hz}}\right)^{2}$ (Yagi \& Seto 2011).

B-DECIGO can be considered as a scaled version of DECIGO, which is build to test the most important technological features of the latter. Still, the scientific goals of this reduced mission remain unchanged, with the main difference being in a lower detector's sensitivity (Sato et al. 2017, Isoyama et al. 2018), and therefore a smaller number of observations are expected. Although the orbital configuration of such a satellite has not been finalized yet, in this paper we assume that B-DECIGO will follow the same trajectory ${ }^{5}$ of its bigger brother, but with a lower noise spectral density given by

$S_{h}^{\mathrm{BD}}(f)=S_{0}\left(1+1.584 \cdot 10^{-2} x^{-4}+1.584 \cdot 10^{-3} x^{2}\right)$,

where and $x=f / \mathrm{Hz}$ and $S_{0}=4.04 \times 10^{-46} \mathrm{~Hz}^{-1}$ (Isoyama et al. 2018).

\section{Appendix $B: S / N$ as a function of the source angles}

The parameter's space spanned by the GW template (1) is rather large, and it is not straightforward in analyzing the dependence of the $\mathrm{S} / \mathrm{N}$ as a function of the source's properties. However, since the luminosity distance $d$ acts as a scale factor, for a specific choice of the component masses, $\rho$ is only determined by the four angles $\zeta=\left(\bar{\theta}_{\mathrm{S}}, \bar{\phi}_{\mathrm{S}}, \bar{\theta}_{\mathrm{L}}, \bar{\phi}_{\mathrm{L}}\right)$. In this Appendix, we investigate how the $\mathrm{S} / \mathrm{N}$ varies in terms of $\zeta$ for prototype BWDs with $m_{1}=m_{1}=1 M_{\odot}$ observed by DECIGO and B-DECIGO. We focus on some specific choices of the azimuthal and polar angles, studying the dependence on the direction of the binary's angular momentum.

Figure B.1 shows the regions, for binaries at $d=100 \mathrm{Mpc}$ detected by DECIGO, where $\rho \geq(200,300)$ for $\bar{\phi}_{\mathrm{S}}=(0, \pi / 4)$ and $\bar{\theta}_{\mathrm{S}}=(\pi / 2, \pi / 4)$. As already noted in Sec. 3 for all the configurations, we obtain very high values for the $S / N$, which are always $\gtrsim 100$ in the entire parameter's space and are modulated by the specific direction of the angular momentum $\hat{L}$. While $\rho$ seems more sensitive to variations of the source azimuth, different choices of the polar angle $\bar{\phi}_{\mathrm{S}}$ lead to qualitatively similar results.

The three panels of Fig. B.2 show the same analysis for BDECIGO and BWDs at $d=100 \mathrm{Mpc}$. Colored islands identify the configurations that are observed with $\rho \geq 10$ and $\rho \geq 8$. The latter represents the detection's threshold fixed in Sec. 3. The shape of the regions resemble the results seen above. However, depending on $\bar{\theta}_{\mathrm{L}}$ and $\bar{\phi}_{\mathrm{L}}$, only specific combinations of these two angles yield binaries observable by the interferometer. These values improve for sources closer to the detector. For example, at $d=50 \mathrm{Mpc}$, all the systems populating the plots in Fig. B.2 would be detectable above the threshold.

\footnotetext{
5 See (Nair \& Tanaka 2018) for a specific example of a geocentric satellite, instead of the one considered in this paper that orbits around a Sun centered frame.
} 

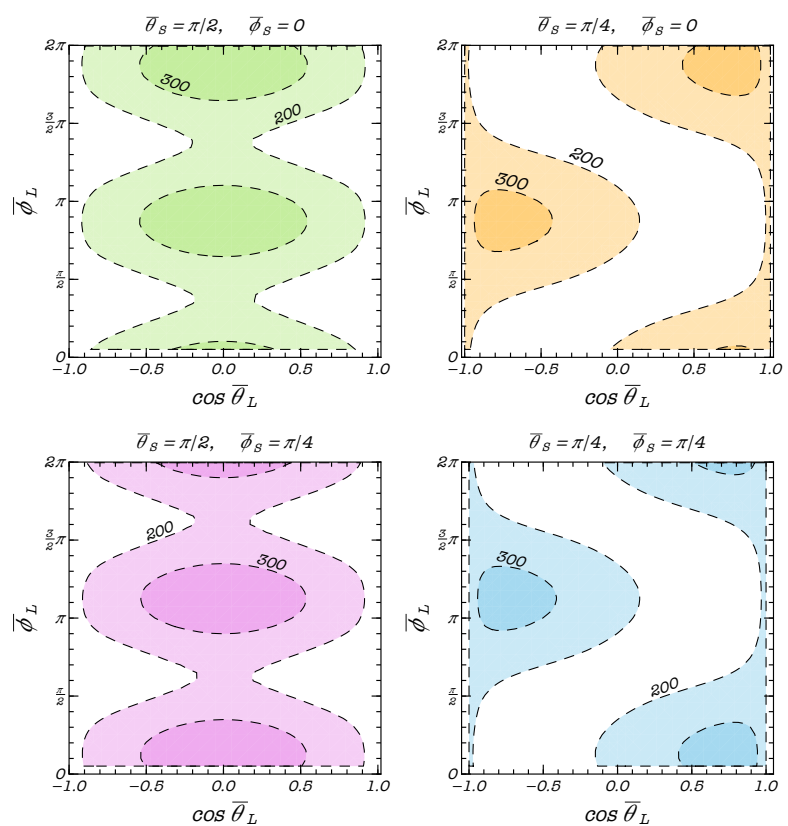

Fig. B.1. Contour regions of S/N for BWD with $m_{1}=m_{2}=1 M_{\odot}$ at $d=100 \mathrm{Mpc}$, observed by DECIGO, as a function of the angles that specify the binary angular momentum, for a fixed source's orientation in the sky, i.e. $\bar{\theta}_{\mathrm{S}}=(\pi / 2, \pi / 4)$ and $\bar{\phi}_{\mathrm{S}}=(0, \pi / 4)$. Dark (light) contours identify regions where $\rho \geq 300(\rho \geq 200)$.
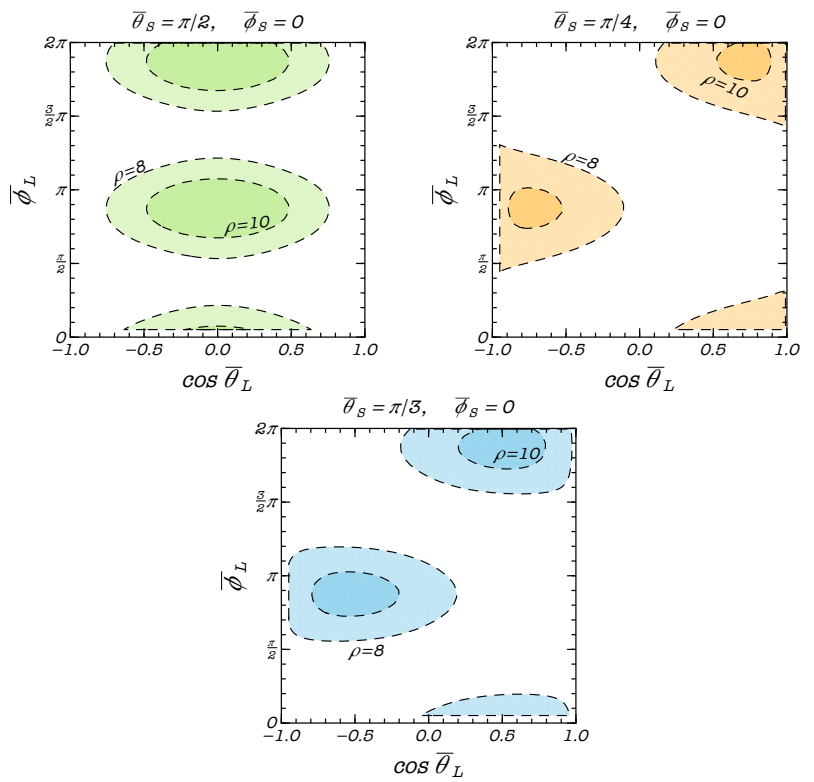

Fig. B.2. Same as Fig. B.1 but for white dwarf binaries detected by BDECIGO at $100 \mathrm{Mpc}$. The shaded regions identify configurations with $\rho \geq 8$ and $\rho \geq 10$. 